\title{
Does the Type of Initial Biopsy Method Determine Re-Excision Rate of Cutaneous Melanoma?
}

\author{
Ramya Vangipuram¹, Mary Ramirez ${ }^{1}$, Yasir Al Abboodi² and Subhasis Misra ${ }^{1 *}$ \\ ${ }^{1}$ Department of Surgery, Texas Tech University Health Sciences Center School of Medicine, Texas, USA \\ ${ }^{2}$ Department of Internal Medicine, Texas Tech University Health Sciences Center School of Medicine, Texas, USA
}

\begin{abstract}
Background: Various biopsy types are used to diagnose melanoma, after which wide local excision (WLE) is the gold standard for treatment. Depending on the final pathology report, further re-excision may be necessary despite an initial, presumably adequate, WLE. In this study, we analyzed the impact of initial biopsy type (shave, punch, or excision) on the management of melanoma.

Methods: A retrospective chart review of 243 patients with clinically node-negative melanoma was completed. Evaluated variables included the initial biopsy type, initial and final peripheral and deep margin status, further reexcision rates, tumor site, and clinician specialty performing the biopsy. Univariate and multivariate analyses tests were performed using SPSS software.

Results: $29.5 \%$ of specimens with both positive peripheral and deep margins underwent further excisions. Overall, $14.6 \%$ of cases had re-excision regardless of initial biopsy type after initial WLE. There was higher rate of re-excision with an initial excision biopsy $(28.6 \%)$ than with initial punch $(13.3 \%)$ or shave biopsies $(11.2 \%)$ Dermatologists, Surgeons, and Primary Care Physicians performed $13 \%, 34 \%$, and $32 \%$ of the excision biopsies respectively. Neither the anatomic location of the tumor nor the survival rates were significantly correlated to the biopsy type, margin status, or re-excision rates.

Conclusion: Both shave and punch biopsies showed high rates of residual tumor in either peripheral or deep margins but this does not translate into a higher re-excision rate. Although the biopsy type is important, the clinical specialty performing the biopsy could be influential. Surprisingly, there was a higher positive peripheral margin than deep margin with shave biopsy.
\end{abstract}

Keywords: Melanoma; Wide local excision; Shave biopsy; Punch biopsy; Re-excision rate

\section{Introduction}

Melanoma is one of the few cancers whose incidence and mortality has been increasing in the U.S. [1-3]. Approximately 77, 000 new cases of melanoma were diagnosed in the United States in 2013 [3]. In the same year, 9,480 deaths were attributed to melanoma [3]. The lifetime risk for the development of melanoma is now 1 in 35 for males and 1 in 54 for females [4]. Suspicious pigmented lesions must be biopsied in order to diagnose melanoma. Although excisional biopsy is the recommended diagnostic procedure for melanoma, clinicians commonly use other methods such as a shave or punch biopsy [2,47]. Moreover, the proportion of cutaneous melanomas diagnosed by non-excisional biopsy techniques is increasing [4]. Wide local excision (WLE) is the gold standard for treatment after an initial diagnosis [2,47]. Thus, a proper initial biopsy is necessary for accurate preoperative tumor staging, which in turn is critical for determining appropriate treatment options for patients with confirmed cancer. These include selection of appropriate resection margins, the need for sentinel lymph node biopsy, and consideration of adjuvant therapy [4]. However, despite presumably sufficient margins after a WLE, re-excision of the tumor site is ultimately dependent on the findings described in the final pathology report. The current management of melanoma begins with performing an initial biopsy for suspicious lesions. For biopsies with confirmed melanoma, the depth of tumor invasion determines the appropriate surgical margins in the consequent WLE. If this excision shows inadequate margin resection, then another re-excision is performed to assure that all margins are free of tumor. In this study, we analyzed the effect of initial biopsy method (shave, punch, or excision) on the management of melanoma, with a particular focus on re-excision rates.

\section{Methods}

After IRB review and approval, 337 patients were initially identified through the Cancer Registry and selected for inclusion. Then, a retrospective chart review of each patient who presented with clinically node-negative melanoma (stages cTI-4N0) from 2008 to 2013 was completed. Patients excluded from the study included those who were lost to follow-up and those without complete pathology reports. Ultimately, 243 eligible patients were included. Evaluated variables included age at time of diagnosis, sex, race, Breslow's depth, presence of ulceration, mitoses on initial or consequent biopsies, Clark's level, location of tumor on the body, initial biopsy type, initial and final peripheral and deep margin status, further re-excision rates, and clinician specialty performing the biopsy. Univariate and multivariate analyses tests were performed using SPSS software.

*Corresponding author: Subhasis Misra, Depertment of Surgery, Texas Tech University Health Sciences Center School of Medicine, 1400 Coulter Street, Amarillo, Texas 79106, USA, Tel: + 806414 9558; Fax: + 806351 3787; E-mail subshasis.misra@ttuhsc.edu

Received September 01, 2014; Accepted March 27, 2015; Published April 05, 2015

Citation: Vangipuram R, Ramirez M, Abboodi YA, Misra S .Does the Type of Initial Biopsy Method Determine Re-Excision Rate of Cutaneous Melanoma? Journal of Surgery [Jurnalul de chirurgie] 2015; 10(4): 267-269 DOI: 10.7438/1584-9341-10-4-5

Copyright: $\odot 2015$ Vangipuram R, et al. This is an open-access article distributed under the terms of the Creative Commons Attribution License, which permits unrestricted use, distribution, and reproduction in any medium, provided the original author and source are credited. 


\section{Results}

Overall, $36(14.6 \%)$ patients underwent re-excision after WLE, irrespective of initial biopsy type. 72 (29.5\%) biopsies with both positive peripheral and deep margins underwent further excisions. 181 (74.5\%) cases were diagnosed by shave biopsy, $59.7 \%$ of which had positive peripheral margin, $34.8 \%$ had positive deep margin, and $19 \%$ had both positive margins. 16 (6.6\%) cases were diagnosed with punch biopsy, $68.8 \%$ of which had a positive peripheral margin, $31.3 \%$ had positive deep margin, and $25 \%$ had both positive margins. 46 (18.9\%) cases were diagnosed with excision biopsy, $39.1 \%$ of which had a positive peripheral margin, $21.7 \%$ had positive deep margin, and $13 \%$ had both positive margins. There was a significantly higher rate of re-excision with initial excision biopsy (28.6\%) than with initial punch $(13.3 \%)$ or initial shave biopsies $11.2 \%(\mathrm{P}=0.01)$. Dermatologists, Surgeons, and Primary Care Physicians did 13\%, 34\% and 32\% of the excision biopsies respectively. Melanoma location and survival rates were not significantly correlated to the biopsy type, margin status, or re-excision rates (Tables 1-3).

\section{Discussion}

Because surgical excision is the primary treatment of biopsyproven melanoma at any site, the initial biopsy is critical in determining the appropriate surgical strategy, including the calculation of optimal surgical resection margins, and the necessity of a sentinel lymph node biopsy. These decisions are largely based on the thickness, ulceration status, and mitotic rate of the primary melanoma [3,6-10]. Re-excision is performed when residual tumor is identified in either peripheral or deep margins. In our study, $29.5 \%$ of cases with both positive peripheral and deep margins underwent further excisions. Overall, $14.6 \%$ cases had re-excision regardless of initial biopsy type after initial WLE.

The majority of cases (74.5\%) in our study were diagnosed by shave biopsy. We found that $59.7 \%$ of all shave biopsies had a positive peripheral margin, $34.8 \%$ had a positive deep margin and $19 \%$ of had both positive peripheral and deep margins. It is surprising that shave biopsies had a higher rate of positive peripheral margins than the rate of positive deep margins. This could be attributed to two plausible theories: the field defect phenomenon and saucerization of the specimen. The field defect phenomenon accounts for microscopic lateral invasion that would not be visible to the clinician performing the biopsy. Typically, melanocytes initially undergo transformation into atypical melanocytes and then further convert into cancerous cells. These changes would not be visible to the unaided eye, and thus could explain the higher positive peripheral margin rate. In addition, the use of a deep scoop shave to obtain tissue leads to saucerization of the biopsy specimen, and renders the shave biopsy with a thicker depth than would be expected with the use of traditional blades for shave biopsies.

Dermatologists, Surgeons, and Primary Care Physicians did 13\%, $34 \%$ and $32 \%$ of the excision biopsies respectively. In our study, dermatologists preferred shave biopsies for suspected melanomas, whereas surgeons and primary care practitioners preferred excision biopsies. Given our finding that initial excision biopsies were associated with the highest rate of re-excision, it is worth investigating if the clinician's field of practice influences the re-excision rate, and ultimately the outcome of complete removal of the tumor.

Some literature suggests that shave biopsies are not recommended when melanoma is suspected because these biopsies may limit the amount of specimen available for adequate pathologic assessment and microstaging, especially in regard to tumor thickness [4-6,11]. However, our data proposes that shave and punch biopsies may be a sufficient means of initial diagnosis because these methods are not associated with a higher rate of re-excision. Even though both of these types of biopsies had positive peripheral and deep margins, the resulting WLE performed was adequate enough to remove the melanoma completely. In addition, shave and punch biopsies are simpler and less expensive to perform, and cosmesis becomes less of a concern.

46 (18.9\%) cases were diagnosed with excision biopsy, 39.1\% of which had a positive peripheral margin, $21.7 \%$ had a positive deep margin, and $13 \%$ had both positive margins. The high positive rate of peripheral margin may be attributed to the use of excision biopsy when the suspicion for melanoma is high, compared to the preference for a shave or punch biopsy when the suspicion for melanoma is low. By the time the patient had presented with a cutaneous lesion that looked suspicious enough to warrant an initial excisional biopsy, the tumor may have already exhibited microscopic lateral spreading, which is not identifiable without a histopathological examination.

$16(6.6 \%)$ cases were diagnosed with punch biopsy, $68.8 \%$ of which had a positive peripheral margin, $31.3 \%$ had positive deep margin, and $25 \%$ had both positive margins. Although punch biopsies may sample enough tissue depth, they do not provide enough information to assess the lateral growth of melanocytic tumors, which is also essential for proper diagnosis [12]. Our findings support the statement that punch biopsies are seldom used in the diagnosis of a suspected melanocytic tumor.

Newer literature suggests that simpler types of initial biopsies may be sufficient for diagnosing and staging melanoma and providing sufficient information for consecutive management plans $[10,11,13$ 15]. A study by Hieken et al, with a similar design to that of ours, found that only $2 \%$ of its patients were diagnosed by excisional biopsy, and only $23 \%$ of patients diagnosed by biopsies with positive deep and lateral margins needed additional cancer treatment after final pathology

Table I: Patient Characteristics.

\begin{tabular}{|c|c|c|}
\hline Characteristics & Patients & $(n=243)$ \\
\hline \multicolumn{3}{|c|}{ Gender } \\
\hline Male & 148 & $60.90 \%$ \\
\hline Female & 95 & $39.15 \%$ \\
\hline \multicolumn{3}{|l|}{ Race } \\
\hline White & 238 & $98.00 \%$ \\
\hline Non-White & 2 & $0.01 \%$ \\
\hline Unknown & 3 & $0.01 \%$ \\
\hline \multicolumn{3}{|c|}{ Age (years) } \\
\hline $10-19$ & 2 & $0.01 \%$ \\
\hline $20-29$ & 6 & $0.02 \%$ \\
\hline $30-39$ & 13 & $0.54 \%$ \\
\hline $40-49$ & 39 & $16 \%$ \\
\hline $50-59$ & 57 & $23.50 \%$ \\
\hline $69-69$ & 52 & $21.40 \%$ \\
\hline $70-79$ & 50 & $20.10 \%$ \\
\hline $80-89$ & 12 & $4.90 \%$ \\
\hline $90-99$ & 1 & $0.00 \%$ \\
\hline Median Age & \multicolumn{2}{|c|}{60} \\
\hline \multicolumn{3}{|c|}{ Stage } \\
\hline 0 & 55 & $22.60 \%$ \\
\hline IA & 81 & $33.30 \%$ \\
\hline IB & 39 & $16.00 \%$ \\
\hline I NOS & 15 & $6.17 \%$ \\
\hline IIA & 9 & $3.70 \%$ \\
\hline IIB & 9 & $3.70 \%$ \\
\hline IIC & 1 & $0.00 \%$ \\
\hline IIIA & 3 & $1.23 \%$ \\
\hline IIIC & 6 & $2.05 \%$ \\
\hline III NOS & 5 & $2.05 \%$ \\
\hline IV & 11 & $4.52 \%$ \\
\hline Unknown & 8 & $3.29 \%$ \\
\hline
\end{tabular}


Table II: Biopsy Characteristics.

\begin{tabular}{|c|c|c|}
\hline Characteristics & Patients & $(n=243)$ \\
\hline \multicolumn{3}{|c|}{ Breslow's Depth (mm) } \\
\hline $0<x<1$ & 129 & $53.09 \%$ \\
\hline $1<x<2$ & 34 & $13.99 \%$ \\
\hline $2<x<4$ & 16 & $6.58 \%$ \\
\hline$>4$ & 11 & $4.12 \%$ \\
\hline Unknown & 53 & $21.81 \%$ \\
\hline \multicolumn{3}{|c|}{ Clark's Level } \\
\hline I & 52 & $21.40 \%$ \\
\hline II & 62 & $25.51 \%$ \\
\hline III & 31 & $12.76 \%$ \\
\hline IV & 46 & $18.93 \%$ \\
\hline V & 5 & $2.05 \%$ \\
\hline Unknown & 47 & $19.34 \%$ \\
\hline \multicolumn{3}{|c|}{ Site of Tumor } \\
\hline Head and Neck & 84 & $34.57 \%$ \\
\hline Trunk & 51 & $20.10 \%$ \\
\hline Upper Extremity & 72 & $29.63 \%$ \\
\hline Lower Extremity & 36 & $14.81 \%$ \\
\hline \multicolumn{3}{|c|}{ Ulceration Present } \\
\hline Initial Biopsy & 78 & $11.52 \%$ \\
\hline Final Biopsy & 24 & $24 \%$ \\
\hline \multicolumn{3}{|c|}{ Mitoses Present } \\
\hline Initial Biopsy & 78 & $32.10 \%$ \\
\hline Final Biopsy & 24 & $9.88 \%$ \\
\hline
\end{tabular}

Table III: Survival Rates.

\begin{tabular}{|c|c|}
\hline Variable & p -Value \\
\hline Location & 0.991 \\
\hline \multicolumn{2}{|c|}{ Margin Status } \\
\hline Positive Peripheral & 0.00 \\
\hline Positive Deep & 0.826 \\
\hline Re-excision & 0.083 \\
\hline
\end{tabular}

at WLE [4]. Moreover, the study discovered that most diagnostic biopsies were margin positive regardless of biopsy technique, and that more than one third of patients had residual melanoma on WLE [4]. These literature findings, along with ours, propose that less invasive techniques such as shave and punch biopsies are an adequate means of accurately diagnosing suspected melanocytic tumors.

\section{Conclusion}

Both shave and punch biopsies showed high rates of residual tumor in either peripheral or deep margins; however, this is not correlated with a higher re-excision rate. Although the biopsy type is important, clinical specialty performing the biopsy could be influential. Surprisingly, there was a higher positive peripheral margin than deep margin with shave biopsy, which could be due to the field defect phenomena in melanoma.

These data suggest that shave or punch biopsies are preferable to excision biopsies for an initial biopsy when a primary cutaneous melanoma is initially suspected. Although they may be associated with a higher likelihood of residual tumor, they were not correlated with an increased risk of re-excision. Also, initial WLE removes a wider area of skin, which can lead to increased healing time, increased cost, and is less cosmetically appealing. The limitations of our study include the biases innate to retrospective chart reviews, including the need to exclude some patients because of incomplete data for analysis. Our study is unique in that it suggests that a simpler, less invasive, and less expensive method of biopsy can provide the same level of information, as does an excisional biopsy, while decreasing the rate of re-excision.

\section{Conflict of interests}

Authors have no conflict of interests to disclose.

\section{References}

1. Lakhani NA, Saraiya M, Thompson TD, King SC, Guy GP Jr (2014) Total body skin examination for skin cancer screening among U.S. adults from 2000 to 2010. Prev Med 61: 75-80.

2. Mills JK, White I, Diggs B, Fortino J, Vetto JT (2013) Effect of biopsy type on outcomes in the treatment of primary cutaneous melanoma. Am J Surg 205: 585-590.

3. American Cancer Society (2013) Cancer facts and figures 2013. Atlanta (GA): American Cancer Society.

4. Heiken TJ, Hernández-Irizarry R, Boll JM, Jones Coleman JE (2013) Accuracy of Diagnostic Biopsy for Cutaneous Melanoma: Implications for Surgical Oncologists. Int J Surg Oncol 2013: 196493.

5. Ott PA, Berman RS (2011) Surgical approach to primary cutaneous melanoma Surg Oncol Clin N Am 20: 39-56.

6. Egnatios GL, Dueck AC, Macdonald JB, Laman SD, Warschaw KE, et al (2011) The impact of biopsy technique on upstaging, residual disease, and outcome in cutaneous melanoma. Am J Surg 202: 771-777.

7. Molenkamp BG, Sluijter BJ, Oosterhof B, Meijer S, van Leeuwen PA (2007) Non-radical diagnostic biopsies do not negatively influence melanoma patient survival. Ann Surg Oncol 14: 1424-1430.

8. Swanson NA, Lee KK, Gorman A, Lee HN (2002) Biopsy techniques. Diagnosis of melanoma. Dermatol Clin 20: 677-680.

9. Zager JS, Hochwald SN, Marzban SS, Francois R, Law KM, et al. (2011) Shave biopsy is a safe and accurate method for the initial evaluation of melanoma. $J$ Am Coll Surg 212: 454-460.

10. Ng JC, Swain S, Dowling JP, Wolfe R, Simpson P, et al. (2010) The Impact of Partial Biopsy on Histopathologic Diagnosis of Cutaneous Melanoma: Experience of an Australian Tertiary Referral Service. Arch Dermatol 146: 234-239.

11. Stell VH, Norton HJ, Smith KS, Salo JC, White RL Jr (2007) Method of biopsy and incidence of positive margins in primary melanoma. Ann Surg Oncol 14: 893-898.

12. Witheiler DD, Cockerell CJ (1992) Sensitivity of diagnosis of malignant melanoma: a clinicopathologic study with a critical assessment of biopsy techniques. Exp Dermatol 1: 170-175.

13. Pflugfelder A, Weide B, Eigentler TK, Forschner A, Leiter U, et al. (2010) Incisional biopsy and melanoma prognosis: facts and controversies. Clin Dermatol 28: 316-318.

14. Rampen FH, van der Esch EP (1980) Biopsy and survival of malignant melanoma. J Am Acad Dermatol 5: 313-320.

15. Austin JR, Byers RM, Brown WD, Wolf P (1996) Influence of biopsy on the prognosis of cutaneous melanoma of the head and neck. Head Neck 18: 107-117. 